\title{
RECENT PROGRESS ON THE MECHANISMS OF EMBRYONIC LENS FORMATION
}

\author{
R. M. GRAINGER, J. J. HENRY,* M. S. SAHA, M. SERVETNICK \\ Charlottesville, USA
}

\begin{abstract}
SUMMARY
Formation of the lens during embryonic development depends on tissue interactions as shown clearly both from teratological data and from extensive experimental analysis. Recent work has, however, altered our view of the importance of particular tissue interactions for lens formation. While earlier work emphasises the role of the optic vesicle in lens induction, more recent studies argue that lens-inducing signals important for determination act before optic vesicle formation. Evidence is given for a four stage model in which ectoderm first becomes competent to respond to lens inducers. It then receives inductive signals, at least in part emanating from the anterior neural plate, so that it gains a lens-forming bias and subsequently becomes specified for lens formation. Complete lens differentiation does require signals from the optic vesicle, and in addition an inhibitory signal from head neural crest may suppress any residual lens-forming bias in head ectoderm adjacent to the lens.
\end{abstract}

Long before the advent of experimental embryology, nineteenth century biologists and physicians surmised that proper development of the lens depended on interactions of the presumptive lens tissue with surrounding tissues, most notably the developing retina. ${ }^{1}$ It was already clearly understood that the lens was derived from ectoderm which overlies the eye rudiment (the relationship of these two tissues just before lens differentiation commences can be seen in Fig. 4). In abnormal human and animal embryos in which the eye cup was detached from the overlying ectoderm, or in the case of anophthalmia, when the eye cup was not present, lenses were also missing. Similar inferences were derived from observations of the large median eye of cyclopean monsters, since these had a single lens in association with the eye, suggesting that the site of lens development was linked to the site of eye formation. ${ }^{1}$

As was pointed out by Spemann in his classic 1901

From: Department of Cell and Structural Biology, University of Illinois, Urbana, Illinois 61801, USA.

Correspondence to: Dr. R. M. Grainger, Department of Biology, Gilmer Hall, University of Virginia, Charlottesville, VA. 22901, USA. study, ${ }^{2}$ however, the correspondence of eye defects with effects on the lens did not allow one to distinguish whether the lens effects were a consequence of disrupting a normal inductive effect of the eye on lens formation, or were due to an abnormality common to both tissues. Spemann resolved the question by ablating the optic rudiment from very young embryos, the first experiment designed to define any embryonic inductive interaction. Not only was the eye subsequently missing in these embryos, but so was the lens, which is derived from ectoderm which was not damaged in the experiment; the necessity of the eye for lens determination was thus established. A second experiment, by Lewis, ${ }^{3}$ helped shape the basic view of lens induction held for many years. Lewis transplanted the optic vesicle beneath non-lens ectoderm, and showed that a lens was found at the ectopic site. From these results it was argued that the eye rudiment is sufficient for lens induction.

Since the turn of the century the proposal that the eye is both necessary and sufficient for lens induction, which was derived from these two experiments and numerous others, has provided a clear framework for thinking about lens determination. Most of these experiments have utilized amphibian embryos, because they are so readily manipulated surgically, though experiments on chick and mouse embryos, and more incomplete information about human development, indicate common developmental mechanisms for all vertebrates. A review of these older experiments indicates that some of them are difficult to interpret because of both technical problems and interpretation of data, ${ }^{4}$ and it is now clear that lens development is a more complex process than suggested by the framework described above. In recent years reinvestigation of this classic problem, ${ }^{5,6,7,8}$ primarily in the amphibian Xeno$p u s$, has led us to a model for lens induction which postulates at least four stages in this process. We find that there is a brief initial period, during gastrulation, in which ectoderm gains the ability, or competence, to respond to lens inducers. After lens induction commences during the competent period there is an intermediate stage in which a 
large region of head ectoderm gains what we term a lensforming bias as a result of early inductive signals. By the time of neural tube formation the lens ectoderm becomes able to differentiate on its own (specification ${ }^{9}$ ), though it will only form a rather rudimentary lens at this point. Finally, the optic vesicle provides continued signals which enhance differentiation of the specified lens tissue while other tissue interactions suppress the lens-forming bias in adjacent regions of head ectoderm.

Before discussing the experimental evidence for this four stage model, a brief introduction to eye and lens development, which again has been most completely described for amphibian embryos, will provide useful background information. At the late blastula stage the regions that will form ectoderm, mesoderm (marginal zone) and endoderm are delineated (Fig. 1) though the differentiation of these regions has not yet begun. During gastrulation, induction of the nervous system (including the eye regions), on the dorsal side of the embryo, is clearly underway, as the dorsal mesoderm comes to lie under the presumptive neural tissue and imparts neuralinducing signals to it (Fig. 2); there is, however, no morphological evidence of neural or eye differentiation at this stage. One can also identify the regions of ectoderm at this stage that will give rise to the lens: they are just outside the presumptive neural ectoderm and close to the presumptive eye regions. By the neural plate stage, the outlines of the nervous system are defined by the neural folds around the edge of the newly formed neural plate, with the presumptive eye regions just inside the anterior portion of the neural plate and the presumptive lens regions just outside of it (Fig. 3). The subsequent folding of the neural plate to form the neural tube results in the juxtaposition of the optic vesicles with the presumptive lens ectoderm (Fig. 4). Shortly after this stage when contact between the optic vesicle and presumptive lens ectoderm has occurred the lens ectoderm begins to differentiate into a recognisable lens vesicle.

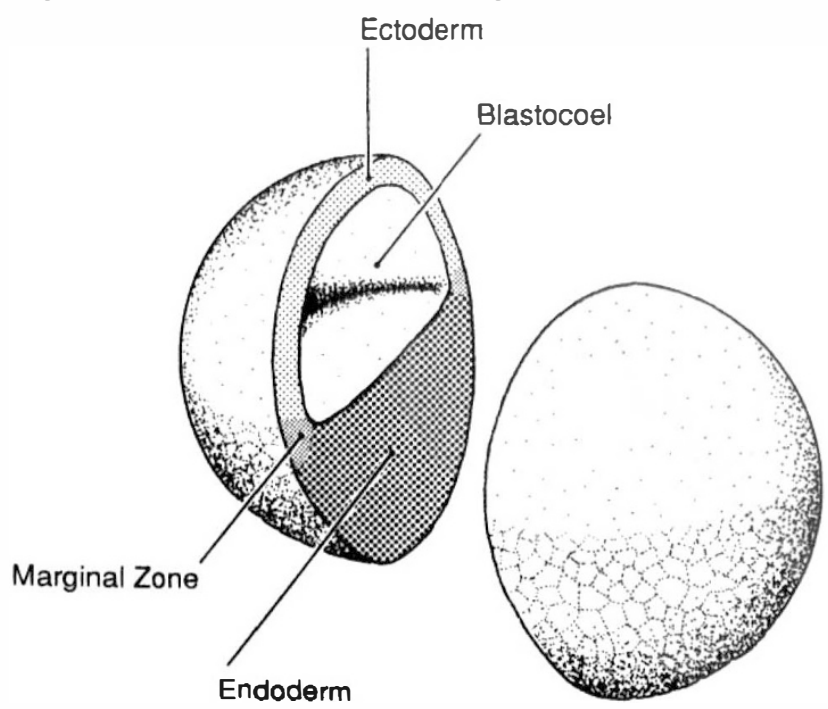

Fig. 1. Late blastula stage. All epidermis, neural tissue and placodal tissues (such as lens, ear and nose) are derived from the ectoderm. At this stage ectoderm is not yet competent for lens formation, however.

\section{PERIOD OF ECTODERMAL COMPETENCE FOR LENS FORMATION}

The initial stage of determination of any tissue responding to inductive signals must involve the acquisition of competence to respond to those signals. Although very little is understood about this important process in any developmental system, the timing of competence does appear to be carefully regulated where it has been examined. ${ }^{8}$ To begin to learn more about this part of the lens induction mechanism we determined the period during which embryonic ectoderm is competent to respond to lens-inducing signals. Ectoderm has periods in which it is competent to form other tissues as well, but what distinguishes the period of lens competence is that it is comparatively quite short, only a few hours during the middle of gastrulation, slightly before the stage shown in Fig. $2{ }^{8}$ Competence was tested in uninduced ectoderm, that is, ectoderm which has not yet been underlain by other tissues (e.g., the entire ectodermal region in Fig. 1, or the small region of ectoderm adjacent to the blastocoel in Fig. 2). To assay competence pieces of this ectoderm were placed into the presumptive lens area of a neural plate stage host (Fig. 3) from which the presumptive lens ectoderm had been removed. As will be discussed below this exposes the ectoderm to the important signals required for lens induction, thus permitting a rigorous assessment of its lens-forming potential. One important prediction of the short competent period is that this defines the time at which lens determination commences.

While early gastrula ectoderm (just slightly after the stage shown in Fig. 1) does not yet have lens competence.

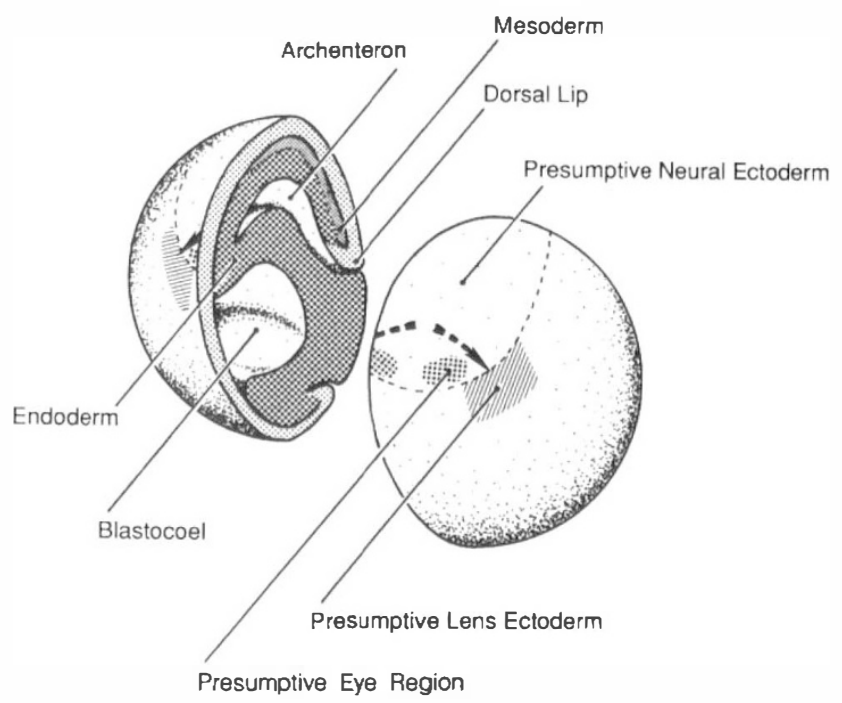

Fig. 2. Mid/late gastrula stage. Lens induction is presumed to be underway at this stage. At this point the lens-forming competence of ectoderm is still high. During this stage the presumptive lens ectoderm first contacts the tissue which has involuted the farthest during gastrulation, the presumptive foregut endoderm, which may act as an early lens inducer. Experiments with Xenopus embryos indicate that an important interaction with the presumptive neural plate and presumptive lens ectoderm occurs at this stage (arrows). The presumptive eye regions are also denoted, and may be the source of this early inductive signal. 


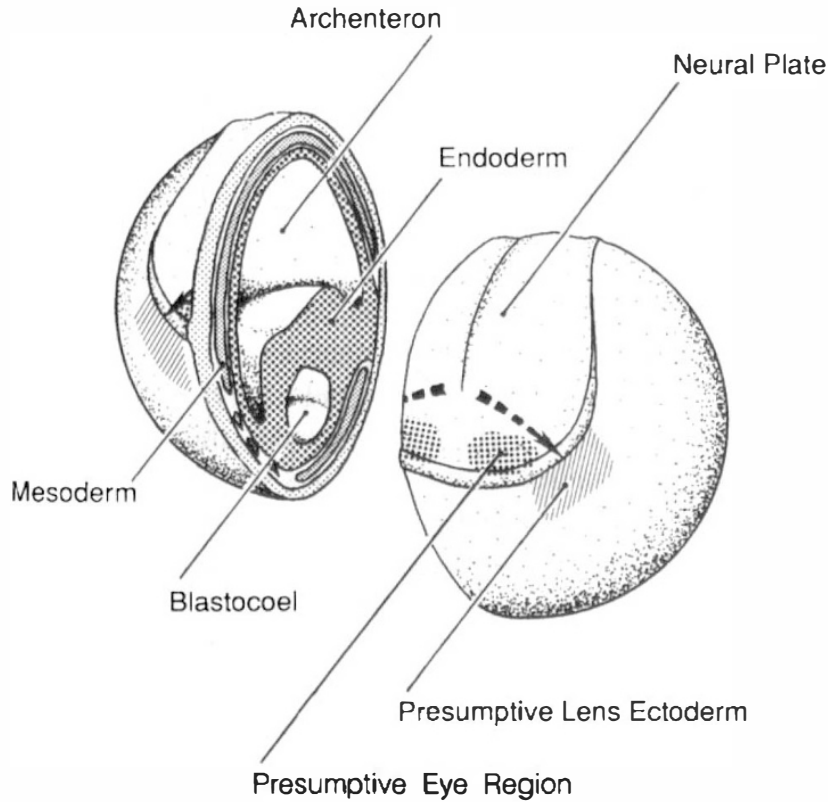

Fig. 3. Neural plate stage. The boundaries of neural tissue are delineated at this stage by the neural folds, with the presumptive eye regions just inside the folds, and the presumptive lens ectoderm just outside of them. At this stage the presumptive lens ectoderm is underlain by mesoderm which contributes to the lens-forming response in Xenopus. The continued inductive influence of the anterior neural plate at this stage is indicated by the arrows.

if it is removed from the embryo and cultured, it gains and loses competence with the same time course as ectoderm in vivo, implying that an autonomous timing mechanism in some way controls the period of competence. While the molecular basis of such a timer is not known, it will certainly be an intriguing problem to analyse in future work.

\section{IS THE OPTIC VESICLE SUFFICIENT FOR LENS INDUCTION?}

Having established that lens competence is restricted to a short period during gastrulation, what tissues are providing the lens-inducing signals at this time? It seems appropriate to begin a discussion of lens-inducing signals with a consideration of the role of the optic vesicle in lens determination, since the classic model for lens induction argued that this is the primary lens inducer. In a formal sense it now seems improbable that this is the case in vivo, based on our recent competence results. Since optic vesicles are only formed at the time of neural tube closure and the competence period is much earlier, during gastrulation, other tissues are implicated as lens inducers at early developmental stages in the embryo.

The question nonetheless remains whether the optic vesicle can, on its own, induce lenses, even if it does not in vivo. While the experiments of Lewis ${ }^{3}$ had suggested that the optic vesicle alone was able to cause lens formation in non-lens ectoderm, a review of this and many similar studies, ${ }^{4}$ and our own recent experimental analyses ${ }^{5,6}$ have raised questions about the general validity of this conclusion and led us to the multiple step model outlined above. We attempted to test Lewis' conclusion, both in the species he used (Rana palustris) and in Xenopus. Our experiments involved determining whether early to mid gastrula ectoderm (not yet underlain by any tissues; slightly later than the stage shown in Fig. 1) would form a lens when placed over the optic vesicle of neural tube stage hosts (as in Fig. 4) from which the presumptive lens ectoderm had been removed. Lenses were often found in these transplants; however, these were not due to induction but to the differentiation of presumptive lens cells that had adhered to the optic vesicle. This was most clearly demonstrated by using lineage tracers (either horseradish peroxidase or a fluoresceinated dextran) to label embryos used for donor tissues; we found that in these cases lenses were invariably derived from host and not donor tissue. Once the optic vesicle has been in contact with the presumptive lens area for several hours, it is very difficult to separate the two tissues cleanly unless proteolytic enzyme treatments are used. Other investigators, as far back as $1907,{ }^{10}$ also noted the difficulty in removing presumptive lens ectoderm from the optic vesicle. If embryos are taken as the neural folds are closing, when the optic vesicle is just approaching the presumptive lens ectoderm, it is possible to isolate the optic vesicle without adhering presumptive lens cells; however, lenses were still not induced in ectoderm placed over these optic vesicles. Although most early studies of amphibian lens induction did not use host and donor marking, and are therefore impossible to interpret, several studies did and these concur with our findings.

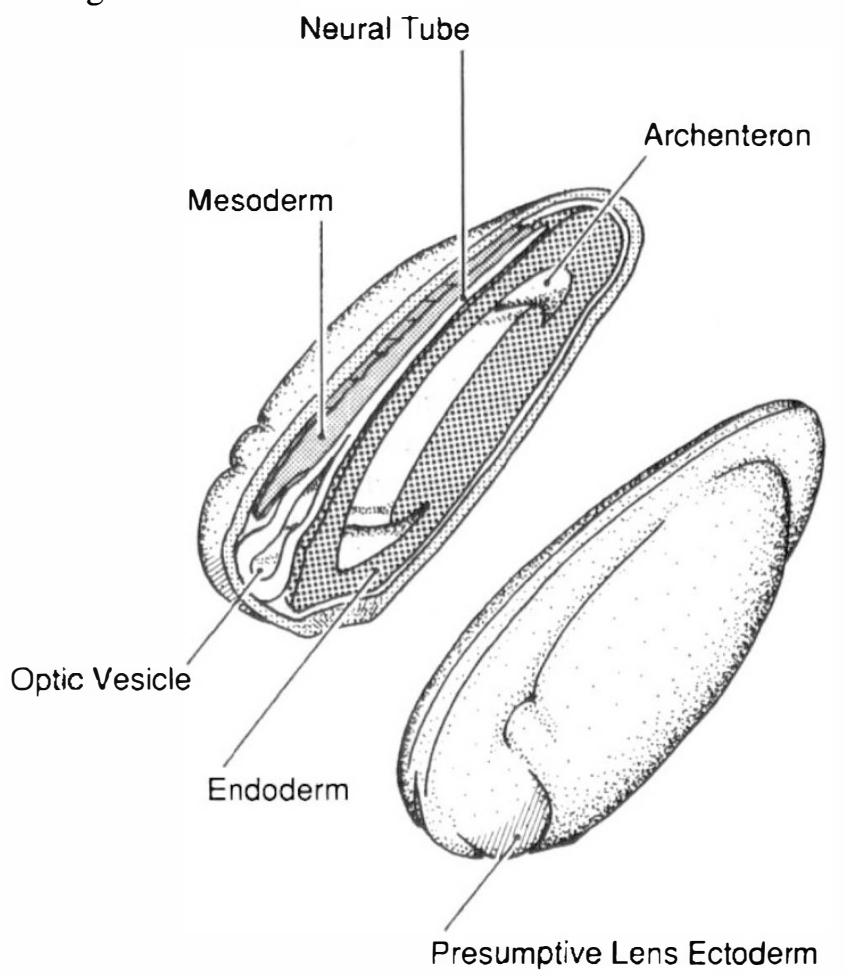

Fig. 4. Neural tube stage. At this time the presumptive lens ectoderm first comes into contact with the newly formed optic vesicles. Inductive interactions between these tissues result in enhanced differentiation of the lens. Surrounding tissues may at this point inhibit any lens-forming bias in ectoderm adjacent to the lens region. 
Our recent results showing that ectoderm has such a short period of lens competence has altered our interpretation of some of these experiments, though not our general conclusions. Because the experiments transplanting ectoderm over the optic vesicle of neural tube stage hosts were done before we had learned that ectoderm has such a short period of competence, no effort was made to control precisely the stages of the grafted ectoderm. It was therefore likely that in some cases the ectoderm was either too young or too old to respond if in fact it could respond to the optic vesicle. We have recently obtained evidence that competent ectoderm, when placed in the presumptive lens area of early neural tube stage hosts, can in about $50 \%$ of the cases form lenses (Grainger and Cook, unpublished). Overall, however, several lines of evidence still support the argument that the optic vesicle alone cannot cause lens induction. First, the potential cell contamination problem noted above, which is likely to have been a concern in many earlier studies concluding that the optic vesicle is sufficient as a lens inducer, still renders these studies uninterpretable. Second, our recent experiments yielding lenses when competent ectoderm was placed over the optic vesicle were done just at the time of neural tube closure, and it is highly likely that other tissue interactions in addition to that with the optic vesicle are still important at these stages. As a more definitive test we have now found that when competent ectoderm is combined with isolated optic vesicles and cultured in vitro no lens induction is found to occur (Grainger, Servetnick and Cook, unpublished).

\section{EARLY TISSUE INTERACTIONS IN LENS INDUCTION}

If the optic vesicle is not sufficient for lens induction, other tissue interactions must play an essential role in the induction process. The results of many studies ${ }^{4}$ have now shown that lens induction can proceed without the optic vesicle. The first of these ${ }^{11}$ was published just a few years after Spemann's original experiment in 1901, and shows the existence of "free" lenses (lenses formed in the absence of eye tissue) in experiments in which the optic rudiment is removed at the neural plate stage. The demonstration of free lenses provides strong evidence for the inductive potency of tissues acting before the optic vesicle. While appearing to contradict Spemann's initial claim that the eye rudiment is essential for lens induction, it has been argued that different species have a different balance between the significance of early and late inducers. $^{12}$

The most extensive studies arguing for a multiplicity of lens-inducing tissues are those of Jacobson ${ }^{13,14}$ who has proposed that three tissues act in sequence to cause lens determination in the newt Taricha torosa. The presumptive foregut endoderm and presumptive heart mesoderm are the tissues which underlie the presumptive lens ectoderm during gastrulation, and are therefore possible sources of early lens-inducing signals. He argues that the optic vesicle then acts as a later inducer. In a series of experiments combining the presumptive lens ectoderm with the various putative inducing tissues he concludes that the foregut endoderm and heart mesoderm are the most potent inducers.

We have recently examined the role of potential early lens inducers in Xenopus ${ }^{7}$ and reached a somewhat different conclusion than Jacobson. Our studies do argue that early inducers are critical for lens formation, and that they act between mid-gastrula and neural tube stages. However, as the source of such signals they implicate the anterior neural plate, which is adjacent to the presumptive lens ectoderm, rather than the tissues which underlie the ectoderm (Figs. 2 and 3). In our studies we did not find any inductive effect of underlying tissues alone. If these tissues are required at all they are only necessary before the end of gastrulation, since we could demonstrate that anterior neural tissue alone is sufficient as a lens inducer after this time. We did, however, find that the mesoderm underlying the presumptive lens area has a potentiating effect on the inducing ability of the anterior neural plate.

At present there is no clear answer as to why our studies differ from those of Jacobson. While it is possible that the different amphibians used in these studies have different lens-inducing mechanisms, there are other differences in the two studies. At the time of Jacobson's studies one had to rely on morphological assays alone, while our recent studies use a very sensitive lens-specific antibody to identify lenses immunohistochemically, a procedure which greatly enhances discrimination of weak but clear lens inductions from non-specific epidermal thickenings that resemble primitive lenses. Further studies on other organisms will be required, however, to achieve a general view of the nature of the early lens-inducing signals.

\section{RESPONSES TO LENS INDUCTION: ESTABLISHMENT OF A LENS-FORMING BIAS IN HEAD ECTODERM AND LENS SPECIFICATION}

By the neural plate stage the presumptive lens ectoderm as well as surrounding head ectoderm has been exposed to lens-inducing factors that result in this ectoderm gaining what we term a lens-forming bias. This is an empirically defined state referring to a decreased threshold of these tissues to respond to lens inducing signals. At the same time these regions of head ectoderm are simultaneously receiving stimuli for forming other tissues derived from ectodermal placodes, e.g. ear and nose, and in general then they might be said to have placodal bias. However, recent studies indicate that at least lens and ear are likely to be induced by independent mechanisms, and we propose that rather than having a common intermediate placodal state, as has been proposed, ${ }^{14}$ head ectoderm is simultaneously receiving independent "biases" for several tissues at once (Gallagher and Grainger, unpublished).

The evidence for acquisition of a lens-forming bias comes from several experiments. In Xenopus the presumptive lens ectoderm at the neural plate stage forms well differentiated lenses at very high frequency (over 
$80 \%$ ) when placed over the optic vesicle of neural tube stage hosts, ${ }^{5}$ a substantially enhanced response over that obtained when gastrula stage competent ectoderm is used in similar transplants (what we consider the baseline response). Between the neural plate stage and neural tube stage large regions of non-lens head ectoderm show evidence of gaining a lens-forming bias since the younger ectoderm does not form lenses when transplanted to neural tube stage hosts while the older ectoderm does (Grainger and Mannion, unpublished).

In chick embryos the lens-forming bias in head ectoderm is even more apparent between the neural plate and neural tube stage. In these embryos one can remove either the presumptive lens ectoderm or non-lens head ectoderm, place it in culture, and it will differentiate into a small crystallin-synthesising lens-like structure ${ }^{15,16}$ and Sullivan et al., unpublished. In this case the bias is so strong that it requires no further tissue interaction at all to initiate lens formation. Clearly in vivo this bias is suppressed, since only the presumptive lens ectoderm forms a lens (possible mechanisms for this suppression are discussed below).

By the neural tube stage in amphibian embryos, just as the optic vesicle is first coming into contact with the presumptive lens ectoderm, it is specified, that is it will differentiate on its own in culture to the extent that it synthesises lens-specific crystallins and has a lens-like morphology. ${ }^{7}$ This point appears to be reached even earlier in chick embryos as noted above. The overall conclusion is that lens specification does not require contact with the optic vesicle, although the lenses that form in such experiments are small and poorly differentiated.

\section{THE ROLE OF THE OPTIC VESICLE AND OTHER TISSUES IN LATER STAGES OF LENS FORMATION}

Although the optic vesicle is not required for lens specification, the optic rudiment at much earlier stages (late gastrula and early neurula) may be critical for lens determination. At this point all that is known is that in Xenopus some portion of the anterior neural plate, possibly the presumptive eye area, appears to be the principal lens inducer at early stages. Further experiments will be required to define the exact region of the neural plate that provides the important signal.

At later stages, however, the optic vesicle clearly does play a role in proper lens differentiation. Fibre cell formation is rarely seen in explants of ectoderm that have not been exposed to the optic vesicle. ${ }^{7}$ It has been shown that neural retina produces factors which stimulate lens differentiation ${ }^{17}$ and that forms of fibroblast growth factor bound in the neural retina can stimulate lens differentiation in culture. ${ }^{18}$ The vitreous humor of the eye has significant levels of insulin-like growth factor, ${ }^{19}$ which also stimulates lens differentiation in culture.

If a strong lens-forming bias is established in large regions of head ectoderm, it is presumably suppressed as these areas subsequently differentiate into other tissues. In the chick one can demonstrate that the same regions of ectoderm that will self-differentiate into lenses when cultured from neural tube stage embryos will not do so from embryos a day older (Sullivan et al., unpublished). From experimental manipulations in amphibian embryos it has been argued that head neural crest cells can inhibit lensforming tendencies ${ }^{20}$ in head ectoderm. In the chick, head mesoderm, some of which may be derived from neural crest, does suppress the ability of head ectoderm to differentiate into lenses. Perhaps one role of the optic vesicle is to prevent contact of neural crest cells with the presumptive lens area, the differentiation of which might otherwise be suppressed. In mutant mouse embryos in which there is poor contact of the optic vesicle and lens ectoderm, and mesodermal cells do infiltrate between these tissues, lens formation is rudimentary or suppressed. ${ }^{21}$ Whether this is due to an inhibitory effect of the mesoderm, blocking of a positive effector derived from the eye rudiment, or both, remains to be established.

\section{SUMMARY AND FUTURE DIRECTIONS FOR STUDY}

The experiments described above provide the basis for the four stage model of lens induction introduced earlier. Initially gastrula ectoderm gains a lens-forming competence for a short period. During this period lens induction is thought to be initiated by a signal or signals that may come from tissues underlying the presumptive lens ectoderm, but recent evidence implicates an important signal arising from presumptive neural tissue adjacent to the presumptive lens ectoderm, possibly directly from the presumptive eye region. Mesoderm underlying the presumptive lens ectoderm during neurula stages does enhance the inducing effects of the neural plate. Early inductive signals result in a strong lens-forming bias in head ectoderm, and finally lead to lens specification, which appears not to require contact with the optic vesicle. Complete lens differentiation requires factors from the optic vesicle, however, and suppression of a lens-forming bias in non-lens head ectoderm appears to involve inhibitory signals from other tissues, possibly neural crest cells.

While the information obtained about the process of lens induction is far more detailed than that available for induction of most tissues in the embryo, there are still important gaps in our understanding of the tissue interactions responsible for lens formation. As is clear from the discussion above, a better definition of the exact sources of both early and late lens induction signals in vivo is still required, and should be feasible by more precise transplant, recombinant and explant experiments. An additional important direction will be to establish the minimum tissue interaction(s) required for activation of lens formation in vitro (e.g. is neural tissue, without any contribution from mesodermal or endodermal tissue, sufficient for lens induction?). This information will be quite useful as studies move towards a more molecular definition of the lens induction process.

The biochemical signals emanating from lens-inducing tissues and the mechanism responsible for activating and 
repressing lens competence will be important areas of study in the future. As we pinpoint the biological nature of these processes the targets for a more mechanistic analysis become clearer. If a single tissue could elicit lens formation from competent ectoderm, it might be conceivable that there would be a single lens-inducing factor which could be purified from that tissue. However, since lens induction requires extended exposure of ectoderm to the inducing tissue or tissues, one might surmise that several factors are required for lens induction. The signal transduction systems responsible for cell type determination in other systems, e.g. vulval cell determination in the nematode $C$. elegans, ${ }^{22}$ appear to involve such multiple signalling mechanisms.

A very general question that remains unsettled is whether lens formation can be elicited from competent gastrula ectoderm (what we consider the basal, uninduced state) solely by interactions with specific lens-inducing tissues, such as the anterior neural plate. It may be necessary in addition to establish first a positional value system for the lens ectoderm (specifying its anterior/posterior and dorsal ventral identity in the embryo). Recent work on embryonic axis formation in Drosophila indicates that establishment of a positional value system by a hierarchy of signal transduction mechanisms is certainly part of the determination mechanism of many tissues during normal development, ${ }^{23}$ and it has been argued that a similar system is likely to act during vertebrate development as well. ${ }^{24}$ Establishing such a positional identity, if required, is likely to involve a multitude of intercellular signalling events as well.

One approach for untangling the signalling mechanisms required for lens induction will involve identification of particular gene products involved in signalling and early responses to induction. As a first step in that direction we have prepared a cDNA library from presumptive eye and lens tissue and begun to identify putative regulatory genes (e.g. homeobox-containing genes) from this library. The challenge remains for the future to determine the functional networks in which such gene products act to control the lens induction process.

The work was supported by research grants from the NIH (EY-05542 and EY-06675) and NSF (DCB9005468) to RMG, traineeship support from NIH to MSS (HD07192), and fellowship support from NIH to JJH (EY-05938) and MS (EY-06173).

Key words: Development, Embryo, Induction, Lens.

\section{REFERENCES}

1. Herbst C: Formative Reize in der Tierischen Ontogenese, Ein Beitrag zum Verständnis der tierischen Embryonalentwicklung. Leipzig: Arthur Georgi. 1901.

2. Spemann H: Über Correlationen in die Entwickelung des Auges. Verh Anat Ges 1901, 15: 61-79.
3. Lewis WH: Experimental studies on the development of the eye in Amphibia. I. On the origin of the lens. Rana palustris. Am J Anat 1904, 3: 505-36.

4. Saha MS, Spann CL, Grainger RM: Embryonic lens induction: More than meets the optic vesicle. Cell Diff and Devl 1989, 28: 153-72.

5. Henry JJ and Grainger RM: Inductive interactions in the spatial and temporal restriction of lens-forming potential in embryonic ectoderm of Xenopus laevis. Devl Biol 1987, 124: $200-14$

6. Grainger RM, Henry JJ, Henderson RH: Reinvestigation of the role of the optic vesicle in embryonic lens induction. Development 1988, 102: 517-26.

7. Henry JJ and Grainger RM: Early tissue interactions leading to embryonic lens formation in Xenopus laevis. Devl Biol 1990, 141: 149-63.

8. Servetnick M and Grainger RM: Changes in neural and lens competence in Xenopus ectoderm: evidence for an autonomous developmental timer. Development 1991, 112: 177-88.

9. Slack JMW: From Egg to Embryo, Second Edition. Cambridge: Cambridge University Press, 1991.

10. Spemann H: Neue Tatsachen zum Linsenproblem. Zool Anzeiger 1907, 31: 379-86.

11. King HD: Experimental studies on the eye of the frog embryo. Wilhelm Roux's Arch Dev Biol 1905, 19: 85-107.

12. Spemann H: Zur Entwicklung des Wirbeltierauges. Zool. Jb. 1912, 32: 1-98.

13. Jacobson AG: The determination and positioning of the nose, lens, and ear. I. Interactions within the ectoderm, and between the ectoderm and underlying tissues. J Exp Zool 1963, 154: 273-83.

14. Jacobson AG: Inductive processes in embryonic development. Science 1966, 152: 25-34.

15. Barabanov VM and Fedtsova NG: The distribution of lens differentiation capacity in the head ectoderm of chick embryos. Differentiation 1982, 21: 183-90.

16. Karkinen-Jääskeläinen M: Permissive and directive interactions in lens induction. J Embryol Exp Morph 1978, 44: 167-79.

17. McAvoy JW: Beta- and gamma-crystallin synthesis in rat lens epithelium explanted with neural retina. Differentiation 1980, 17: 85-91.

18. McAvoy JW and Chamberlain CG: Fibroblast growth factor (FGF) induces different responses in lens epithelial cells depending on its concentration. Development 1989, 107: 221-28.

19. Beebe DC, Silver MH, Belcher KS, Van Wyk JJ, Svoboda ME, Zelenka PS: Lentropin, a protein that controls lens fiber formation, is related functionally and immunologically to the insulin-like growth factors. Proc Nat Acad Sci USA 1987, 84: 2327-30.

20. von Woellwarth C: Die Rolle des Neuralleistenmaterials und der Temperatur bei der Determination der Augenlinse. Embryologia 1961, 6: 219-42.

21. Silver $\mathbf{J}$ and Hughes FW: The relationship between morphogenetic cell death and the development of congenital anophthalmia. J Comp Neurol 1974, 157: 281-302.

22. Horvitz HR and Sternberg PW: Multiple intercellular signalling systems control the development of the Caenorhabditis elegans vulva. Nature 1991, 351: 535-41.

23. Ingham PW: The molecular genetics of embryonic pattern formation in Drosophila. Nature 1988, 335: 25-34.

24. Martin P and Lewis J: From flies to mice and back again Curr Biol 1991, 1: 33-5. 